\title{
PREVALÊNCIA DE ENTEROPARASITOSES EM CASOS DIAGNOSTICADOS NO MUNICÍPIO DE JOÃO PINHEIRO-MG
}

\author{
Thiago Braga da Silva \\ Taciano dos Reis Cardoso \\ Daniela De Stefani Marquez \\ Hugo Christiano Soares Melo \\ Ana Carolina Nascimento Tirapelli \\ Márden Estêvão Mattos Júnior²
}

\section{RESUMO}

Introdução: As enteroparasitoses são definidas como parasitos que vivem no trato gastrointestinal e são classificadas como um dos principais problemas de saúde pública presentes nos países em desenvolvimento. O estudo teve como objetivo, avaliar e comparar com a literatura, a prevalência de enteroparasitoses em pacientes atendidos pelo laboratório municipal de análises clínicas da cidade de João Pinheiro- MG, entre o período de janeiro de 2010 a dezembro de 2010. Métodos: Neste período, foram diagnosticados 510 casos positivos através do exame parasitológico das fezes, e do total destes casos, foram observados $18(3,5 \%)$ resultados para helmintos e $467(91,6 \%)$ resultados positivos para protozoários intestinais. Os protozoários encontrados com maior frequência foram a Entamoeba coli com 254 (49,8\%) amostras positivas, seguido por Giardia lamblia com 121 $(23,7 \%)$ amostras positivas e Endolimax nana com $92(18,0 \%)$ amostras positivas. Entre os helmintos, o Enterobius vermiculares aparece como principal causa, com $7(1,4 \%)$ amostras positivas. Conclusões: As análises dos resultados mostraram que entre os parasitos analisados neste período, foi observada uma maior prevalência entre os protozoários comensais. A espécie Giardia lamblia teve destaque entre os parasitos considerados patogênicos, causador de complicações intestinais em crianças e pacientes imunossuprimidos, principalmente. A importância do estudo e análise da prevalência das enteroparasitoses no município localizado na região noroeste de Minas Gerais é um indicador para o conhecimento das condições sanitárias locais, possibilitando a implementação de melhorias na região.

Palavras Chave: Enteroparasitoses, Exame parasitológico de fezes, saneamento básico.

\section{ABSTRACT}

2 Endereço eletrônico de contato: mardenbiomed@hotmail.com

Volume 2, Número 1 - Maio, 2016. 
Introduction: The enteroparasitosis are defined as parasites that live in the gastrointestinal tract and are classified as a major public health problems present in the developing countries. The study aimed to evaluate and compare to the literature, the prevalence of intestinal parasites in patients attended by municipal clinical laboratory in the city of João Pinheiro- MG, between the period January 2010 to December 2010. Methods: during this time, 510 positive cases were diagnosed by parasitological examination of stools, and the total of these cases, $18(3,5 \%)$ results for helminths were observed $(3.5 \%)$ and $467(91.6 \%)$ tested positive for intestinal protozoa. Protozoa found most frequently were Entamoeba coli with 254 (49.8\%) positive samples, followed by Giardia lambia with 121 (23,7\%) positive samples and Endolimax nana with $92(18,0 \%)$ positive samples. Among the helminths, the Enterobius vermiculares appears as the main cause, with $7(1.4 \%)$ positive samples. Conclusions: the analyzes of the results show that among parasites analyzed during this period, a higher prevalence was observed between commensal protozoa. The Giardia lamblia species stood out among the considered pathogenic parasites, causing intestinal complications in children and immunosuppressed patients, mostly. The importance of the study and analysis of the prevalence of intestinal parasites in the city located in the northwest of Minas Gerais it is an indicator for the knowledge of local health conditions, enabling the implementation of improvements in the region.

Keywords: Enteroparasitosis, Parasitological Stool examination, basic sanitation.

\section{INTRODUÇÃO}

A ocorrência de enteroparasitoses em determinados municípios brasileiros é reflexo de uma desigualdade social presente em regiões menos desenvolvidas, que sofrem com a ausência de saneamento e educação sanitária, fato este, intimamente relacionado à falta de distribuição de renda. As infecções intestinais por parasitos são uma das maiores responsáveis pela morbimortalidade em países em desenvolvimento, principalmente aqueles com características tropicais onde o índice de desenvolvimento humano (IDH) em muitos casos é considerado insuficiente. ${ }^{(1)}$ No entanto, as infecções intestinais são doenças consideradas negligenciadas e grande parte dos países atingidos não possui um planejamento sócio educacional efetivo.

São vários os fatores ambientais facilitadores de infecções que estão claramente presentes no âmbito das comunidades, entre eles a ausência de água de boa qualidade e Volume 2, Número 1 - Maio, 2016. 
de fossas, dejetos e detritos a céu aberto, solo úmido, altas temperaturas, grande proliferação de insetos e dificuldade de acesso aos serviços públicos de saúde.(2)

As crianças estão mais expostas à contaminação em função do desconhecimento dos princípios básicos de higiene e da maior exposição a partir do intenso contato com o solo, que funciona como um referencial lúdico em torno do qual desenvolvem uma série de jogos e folguedos. Postula-se para uma série de parasitos, particularmente para o $\mathrm{A}$. lumbricoides, que o decréscimo na ocorrência com o passar da idade, ou seja, as baixas taxas de incidência e prevalência em adultos estariam condicionadas não só a uma mudança de hábitos, mas também ao desenvolvimento de imunidade progressiva e duradoura contra tais parasitos. ${ }^{(3)}$

As parasitoses intestinais estão entre os patógenos mais frequentes encontrados em seres humanos. Dentre os helmintos, os mais frequentes são os nematelmintos Ascaris lumbricoides e Trichuri trichiuria e os ancilostomídeos, Necator americanus e Ancylostoma duodenale. Dentre os protozoários, destacam-se Entamoeba hystolitica e Giardia duodenalis. (4)

CONCEIÇÃO et al.(5) estimam que $60 \%$ das infecções por enteroparasitoses estão associadas à deficiências nutricionais, principalmente carência de ferro e vitaminas, além disso, aproximadamente $30 \%$ da mortalidade mundial está correlacionada à doenças de veiculação hídrica.

Os sintomas após a infecção por parasitos são característicos, e as manifestações clínicas são usualmente proporcionais à carga parasitária, à patogenicidade, à virulência das populações de parasitos e à resposta imune do hospedeiro. Alguns indivíduos podem manifestar sintomas mais graves, como os imunodeficientes, as gestantes e os desnutridos, principalmente as crianças $^{(6)}$, ao passo que, a maioria das infecções em adultos imunocompetentes, são consideradas assintomáticas.

Portanto, para um diagnóstico adequado, se faz necessária a realização de uma anamenese completa, exame físico e exames laboratoriais. A anemia, muitas vezes observada durante casos de enteroparasitoses pela perda de sangue no local de fixação do verme adulto, é classificada como anemia ferropriva, afeta cerca de 1,3 bilhões de pessoas no mundo, com alta prevalência e mortalidade em crianças e gestantes. A anemia em crianças está associada com o aumento na mortalidade, retardo no crescimento, retardo no desenvolvimento motor, redução no rendimento escolar e um prejuízo no sistema imune.(7)

Volume 2, Número 1 - Maio, 2016. 
É necessário também, que ocorra mais informativos a população, a fim de conscientizar estes, sobre o risco das parasitoses, e de prevenir as mesmas, assim como também realizar a melhora do saneamento básico da cidade.

Vários programas têm sido dirigidos para o controle das parasitoses intestinais em diferentes países, mas, infelizmente, constata se um descompasso entre o êxito alcançado nos países mais desenvolvidos e aquele verificado nas economias mais pobres. Há que se considerar, portanto, que além da melhoria das condições socioeconômicas e de infraestrutura geral, o engajamento comunitário é um dos aspectos fundamentais para a implantação.(3)

Devido à grande importância epidemiológica de estudos parasitológicos, este trabalho teve o intuito de caracterizar a prevalência das enteroparasitoses nos indivíduos diagnosticados no ano de 2010 no município de João Pinheiro, localizado a noroeste de Minas Gerais, correlacionando-o ao desenvolvimento sócio econômico da população e região.

\section{MATERIAIS E MÉTODOS}

Para o desenvolvimento deste trabalho foram utilizadas 510 amostras fecais de indivíduos cadastrados no laboratório de análises clínicas do hospital municipal de João Pinheiro-MG no período de janeiro a dezembro de 2010.

Analisou-se um total de 510 resultados de exames coprológicos, sendo 228 resultados positivos para o gênero masculino e 282 resultados positivos para o gênero feminino com faixa etária entre 0 e 89 anos de idade.

O método utilizado para análise das amostras fecais foi o direto a fresco indicado em casos de fezes diarreicas e sedimentação espontânea de Hoffmann, devido à sua boa abrangência a um grande número de helmintos e protozoários.

\section{RESULTADOS}

Volume 2, Número 1 - Maio, 2016. 
As análises realizadas em amostras fecais de ambos os gêneros evidenciaram uma superioridade na positividade de amostras do gênero feminino $(282 / 55,1 \%)$ em relação ao gênero masculino (228/44,9\%) (Tabelas 1 e 2).

Os resultados demonstraram uma incidência maior $(p<0,005)$ de infecções causadas por protozoários nos gêneros masculino (210/43,3\%) e feminino (257/53,0\%) em relação à infecções por helmintos, com 11 (2,3\%) amostras positivas no gênero feminino e 7 $(1,44 \%)$ no gênero masculino (Tabelas 1 e 2$)$.

Do total de 510 amostras positivas, 25 (4,9\%), foram caracterizadas como biparasitadas. O gênero feminino apresentou 14 (2,7\%) amostras biparasitadas e o gênero masculino $11(2,1 \%)$, não havendo diferença significativa entre os gêneros, porém, com relevância estatística $(p<0,005)$ quando comparado às amostras parasitadas apenas por um parasito (Tabelas 1 e 2 ).

Em relação a frequência de parasitos intestinais, as infecções por protozoários apresentaram uma prevalência significativa $(p<0,001)$ em relação à helmintos tanto no gênero feminino $(257 / 91,1 \%)$ quanto no masculino $(210 / 92,1 \%)$. De acordo com as faixas etárias, a maior prevalência por protozoários foi evidenciada em indivíduos entre 0 e 9 anos tanto no gênero feminino $(82 / 31,9 \%)$, quanto no gênero masculino $(92 / 43,8 \%)$. As faixas etárias de 10 a 19 anos $(57 / 20,2 \%)$ e 40 a 59 anos $(56 / 19,8 \%)$ no gênero feminino evidenciaram uma alta prevalência de infecções por protozoários (Tabela 1 e 2).

Volume 2, Número 1 - Maio, 2016. 


\begin{tabular}{cccccc}
\hline IDADE & FEMININO & HELMINTOS & PROTOZOÁRIOS & BIPARASITADOS & FEMININO \% \\
\hline 0 a 9 & 90 & 2 & 82 & 6 & 31,9 \\
10 a 19 & 57 & 3 & 51 & 3 & $\mathbf{2 0 , 2}$ \\
20 a 29 & 32 & 2 & 28 & 2 & 11,3 \\
30 a 39 & 32 & 0 & 31 & 1 & 11,3 \\
40 a 59 & 56 & 2 & 54 & 0 & 19,8 \\
60 a 79 & 14 & 1 & 11 & 2 & 4,9 \\
80 a 89 & 1 & 1 & 0 & 0 & 0,35 \\
Total & 282 & 11 & 257 & 14 & 100 \\
\hline
\end{tabular}

Tabela 1. Frequência de parasitos intestinais de acordo com a faixa etária no gênero feminino.

\begin{tabular}{cccccc}
\hline IDADE & $\begin{array}{c}\text { MASCULIN } \\
\text { O }\end{array}$ & $\begin{array}{c}\text { HELMINTO } \\
\text { S }\end{array}$ & $\begin{array}{c}\text { PROTOZOÁRIO } \\
\text { SIPARASITADO }\end{array}$ & $\begin{array}{c}\text { MASCULIN } \\
\text { O \% }\end{array}$ \\
\hline 0 a 9 & 100 & 3 & 92 & 5 & 43,8 \\
10 a 19 & 57 & 1 & 51 & 5 & $\mathbf{2 5 , 0}$ \\
20 a 29 & 12 & 0 & 12 & 0 & 5,3 \\
30 a 39 & 13 & 1 & 12 & 0 & 5,7 \\
40 a 59 & 31 & 1 & 30 & 0 & 13,5 \\
60 a 79 & 14 & 1 & 12 & 1 & 6,1 \\
80 a 89 & 1 & 0 & 1 & 0 & $\mathbf{0 , 4 3}$ \\
Total & $\mathbf{2 2 8}$ & 7 & $\mathbf{2 1 0}$ & 11 & $\mathbf{1 0 0}$ \\
\hline
\end{tabular}

Tabela 2. Frequência de parasitos intestinais de acordo com a faixa etária no gênero masculino.

Volume 2, Número 1 - Maio, 2016. 


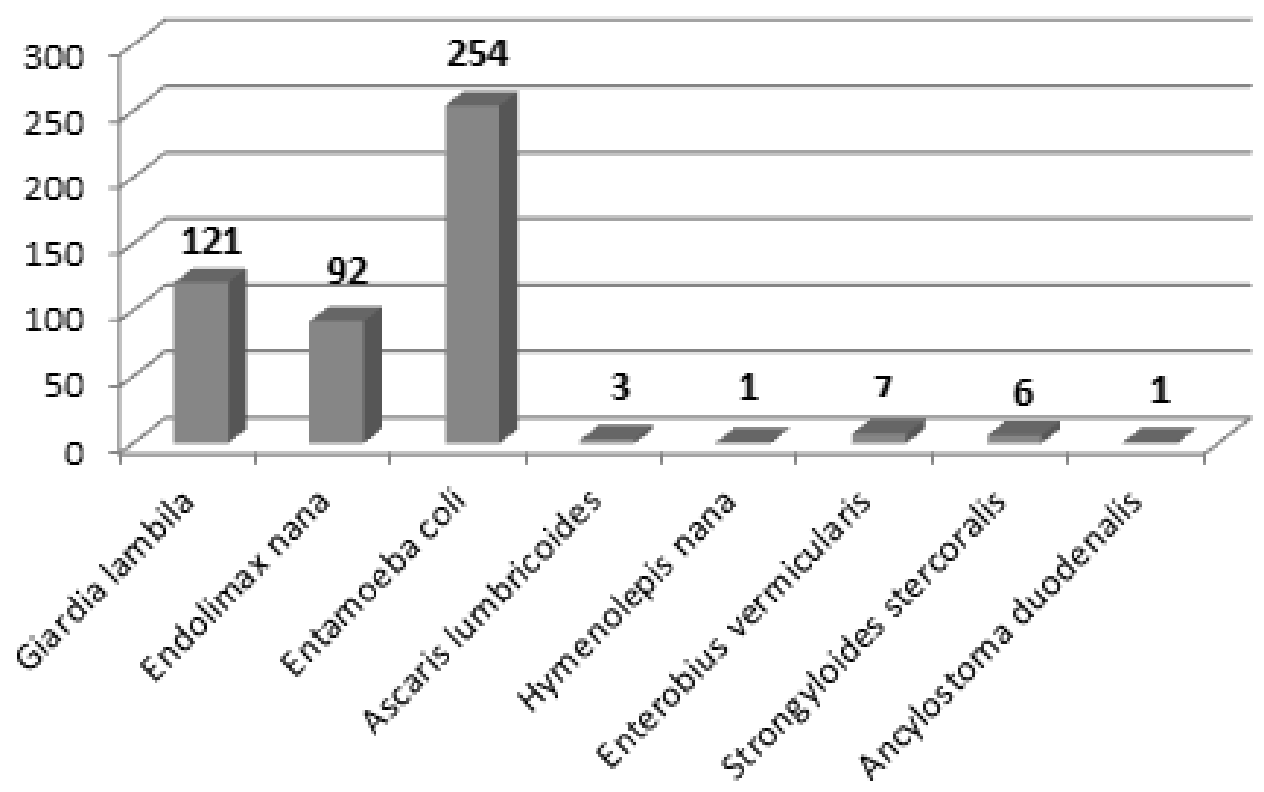

Figura 1: Distribuição amostral da positividade parasitológica após a análise pela sedimentação espontânea de Hoffmann.

\begin{tabular}{lcc}
\hline \multicolumn{1}{c}{ Parasitos } & Amostras Positivas & Frequência \\
\hline Giardia lambila & 121 & $23,7 \%$ \\
Endolimax nana & 92 & $18,0 \%$ \\
Entamoeba coli & 254 & $49,8 \%$ \\
Ascaris lumbricoides & 3 & $0,6 \%$ \\
Hymenolepis nana & 1 & $0,2 \%$ \\
Enterobius vermicularis & 7 & $1,4 \%$ \\
Strongyloides stercoralis & 6 & $1,2 \%$ \\
Ancylostoma duodenalis & 1 & $0,2 \%$ \\
\hline
\end{tabular}

Tabela 3. Distribuição de parasitos por espécie em amostras analisadas positivas.

Volume 2, Número 1 - Maio, 2016. 


\section{DISCUSSÃO}

As parasitoses representam um grande problema de saúde pública, uma vez que atinge em maior prevalência as camadas carentes. Outros fatores relacionados às parasitoses são as condições nutricionais do hospedeiro, higiene pessoal e coletiva, e mecanismo de escape do parasito contra o sistema imunológico. Esses foram os fatores predominantes nos estudos realizados pelos autores dos artigos pesquisados, onde ambos os autores correlacionaram condições sociais e sanitárias com o desenvolvimento das parasitoses.

$\mathrm{Na}$ maioria dos artigos pesquisados foram discutidos que a terapêutica utilizada durante as infecções intestinais, é realizada antes do exame protoparasitológico, o que diminuía as infecções, porém, a recorrência é observada devido ao desenvolvimento sanitário precário. Das amostras analisadas em João Pinheiro os parasitos intestinais foram o de maior enfoque, uma vez que a associação com os fatores já mencionados anteriormente no quesito condições sociais, econômicas e ambientais são os principais responsáveis pelo desenvolvimento dessas parasitoses.

COELHO et al. ${ }^{(8)}$, selecionaram 10 comunidades pré-escolares de regiões periféricas da cidade de Sorocaba, que apresentaram alta incidência de giardíase. O que comprova os dados encontrados nas amostras em João Pinheiro, sendo que ocorreu uma alta incidência de Giárdia sp principalmente no público de 0 a 9 anos. Essa prevalência na incidência de giardíase em crianças foi observada em várias literaturas.

As crianças estão mais expostas à contaminação em função do desconhecimento dos princípios básicos de higiene e da maior exposição a partir do intenso contato com o solo, que funciona como um referencial lúdico em torno do qual desenvolvem uma série de jogos e folguedos. Postula-se para uma série de parasitos, particularmente para o $A$. lumbricoides, que o decréscimo na ocorrência com o passar da idade, ou seja, as baixas taxas de incidência e prevalência em adultos estariam condicionadas não só a uma mudança de hábitos, mas também ao desenvolvimento de imunidade progressiva e duradoura contra tais parasitos. ${ }^{(3)}$

Fazendo um paralelo com os resultados obtidos por diferentes autores pode se observar que as frequências dos parasitas patogênicos Giardia intestinalis (23,7\%), Ascaris lumbricoides $(0,6 \%)$, Enterobius vermicularis $(1,4 \%)$ e Strongyloides stercoralis $(1,2 \%)$

Volume 2, Número 1 - Maio, 2016. 
encontrados na cidade de João Pinheiro, como um todo, corroborando àqueles verificados em outros artigos.

Os parasitas mais frequentemente encontrados em diversos estudos são o Ascaris lumbricoides e Giardia lamblia coincidindo com os nossos achados. Devido à alta prevalência e a frequência de reinfecções, a ascaridíase representa, um importante problema de saúde pública, em consequência da desnutrição e do atraso no crescimento que podem ocasionar. A giardíase pode apresentar-se de forma aguda, principalmente como diarreia, subaguda ou assintomática. Mesmo entre os assintomáticos, obtém-se frequentemente evidência de má-absorção intestinal, prejudicando assim o estado nutricional. ${ }^{(9)}$

Para Tomkins ${ }^{(10)}$, a prevalência de Giardia lamblia um dos parasitas mais encontrados no estudo e o Ascaris lumbricoides, são parasitas que podem interferir no crescimento se estiverem presentes em grande número ou se a ingestão alimentar e as condições gerais de saúde do hospedeiro forem precárias.

É importante também citar os parasitas não patogênicos, sendo Endolimax nana (18 \%) e Entamoeba coli (49,8\%), pois mesmo não causando dano ao organismo elas podem indicar a presença de outros parasitas como foi observado presença de amostras biparasitadas.

A prevalência de enteroparasitoses mostrou-se semelhante nos dois sexos, mas variou intensamente com a idade. Dos artigos analisados somente HURTADOGUERRERO(11) apresentaram maior incidência em população idosa estudo realizado na cidade de Nova Olinda do Norte, Estado do Amazonas - Brasil, o que é necessário um esforço das autoridades governamentais para implementar medidas preventivas e curativas que permitam a melhoria do nível de vida desses idosos, considerando a grave repercussão que esses parasitas tem no estado nutricional e na saúde dos parasitados de baixa renda.

MACEDO(12) retrata que para melhor resultado seria necessário o exame parasitológico de várias amostras do mesmo individuo em dias alternados. O que não foi possível realizar nesse presente estudo.

\section{CONSIDERAÇÕES FINAIS}

Volume 2, Número 1 - Maio, 2016. 
O parasitismo intestinal ainda se constitui um dos mais sérios problemas de Saúde Pública no Brasil, principalmente pela sua correlação com o grau de desnutrição das populações, afetando especialmente o desenvolvimento físico, psicossomático e social de escolares. Embora haja uma vasta literatura sobre a importância das enteroparasitoses para a Saúde Pública, e especialmente, em relação a escolares, pouca atenção tem sido dada ao assunto, nos programas de formação.

As atitudes educativas são válidas, mas precisam ser integradas a um processo contínuo de educação e controle das enteroparasitoses. A maioria das doenças transmitidas por alimentos está ligada aos hábitos precários de higiene pessoal e doméstica dos manipuladores, à higienização e ao controle ambiental, dentre outros.

Algumas maneiras utilizadas para se garantir a qualidade higiênico-sanitária dos alimentos são a realização de programas de educação continuada para os manipuladores de alimentos, a realização semestral de exames parasitológicos desses indivíduos e o fortalecimento do sistema de vigilância sanitária para fiscalização de alimentos oferecidos para a população, incluindo uma legislação adequada.

No Brasil, apesar da relevância e da atualidade do problema, são poucos os trabalhos avaliando a ocorrência de enteroparasitoses em manipuladores de alimentos.

Indica-se que as práticas educacionais quando bem aplicadas levam as pessoas a adquirirem os conhecimentos para prevenção de parasitoses, alcançando objetivos propostos e evidenciando o valor da orientação pedagógica para a conscientização da população.

\section{REFERÊNCIAS}

1. Mezzari A, Perin C, Santos Junior SA, Bernd LA, Di Gesu G. [Airborne fungi and sensitization in atopic individuals in Porto Alegre, RS, Brazil]. Revista da Associação Medica Brasileira. 2003;49(3):270-3.

Volume 2, Número 1 - Maio, 2016. 
2. Fontbonne A, Freese-De-Carvalho E, Acioli MD, Sa GA, Cesse EA. [Risk factors for multiple intestinal parasites in an indigenous community of the State of Pernambuco, Brazil]. Cadernos de saúde pública. 2001;17(2):367-73.

3. Ludwig KM, Frei F, Alvares Filho F, Ribeiro-Paes JT. Correlação entre condições de saneamento básico e parasitoses intestinais na população de Assis, Estado de São Paulo. Rev Soc Bras Med Trop. 1999;32(5):8.

4. Ferreira MU, Ferreira CDS, Monteiro CA. Tendência secular das parasitoses intestinais na infância na cidade de São Paulo (1984-1996). Rev Saúde Pública. 2000;34(6):9.

5. Conceição JS, Fernando LVF, Carvalho TF, Carvalho KB, Pereira EB, Carolina AFLM. Parasitismo por Ascaris lumbricoides e seus aspectos epidemiológicos em crianças do Estado do Maranhão. Revista Sociedade Brasileira de Medicina Tropical. 2011;44(1):2.

6. Pereira VTF. Fatores associados à prevalência de enteroparasitoses em crianças de 0 a 4 anos do município de Patos de Minas, MG. Franca - SP: Universidade de Franca; 2007.

7. Silva IS, Borba E, Cardoso AP, Shirmer H, Wagner S, Rosso R, et al. Avaliação da Prevalência de Enteroparasitoses e sua Associação a Presença de Anemia e/ou Carência Nutricional em Escolares do Município de Flores da Cunha/RS. Revista News Lab. 2011;107:9.

8. Coelho LM, Oliveira SM, Milman MH, Karasawa KA, Santos R. [Detection of transmissible forms of enteroparasites in water and vegetables consumed at schools in Sorocaba, Sao Paulo state, Brazil]. Revista da Sociedade Brasileira de Medicina Tropical. 2001;34(5):47982.

9. Ferreira HdS, Assunção MLd, Vasconcelos VSd, Melo FPd, Oliveira CGd, Santos TdO. Saúde de populações marginalizadas: desnutrição, anemia e enteroparasitoses em crianças de uma favela do "Movimento dos Sem Teto", Maceió, Alagoas. Rev Bras Saude Mater Infant. 2002;2(2):8.

10. Tomkins AM, Dunn DT, Hayes RJ. Nutritional status and risk of morbidity among young Gambian children allowing for social and environmental factors. Transactions of the Royal Society of Tropical Medicine and Hygiene. 1989;83(2):282-7.

Volume 2, Número 1 - Maio, 2016. 
11. Hurtado-Guerrero AF, Alencar FH, Hurtado-Guerrero JC. Ocorrência de enteroparasitas na população geronte de Nova Olinda do Norte Amazonas, Brasil. Acta Amaz. 2005;35(4):3.

12. Macedo H. Prevalência de parasitos e comensais intestinais em crianças de escolas da rede pública municipal de Paracatu (MG). Revista Brasileira de Análises Clínicas.

2005;37(4):4.

Volume 2, Número 1 - Maio, 2016. 